\title{
An Analytical Model to Evaluate the Radiated Power Spectrum of a Multipactor Discharge in a Parallel-Plate Region
}

\author{
Edén Sorolla, Sergio Anza, Benito Gimeno, Member, IEEE, Antonio M. Pérez, Carlos Vicente, Jordi Gil,
} Francisco J. Pérez-Soler, Student Member, IEEE, Fernando D. Quesada, Member, IEEE, Alejandro Álvarez, Senior Member, IEEE, and Vicente E. Boria, Senior Member, IEEE

\begin{abstract}
This paper is aimed at studying the electromagnetic radiation pattern of a multipactor discharge occurring in a parallel-plate waveguide. The proposed method is based on the Fourier expansion of the multipactor current in terms of timeharmonic currents radiating in the parallel-plate region. Classical radiation theory combined with the frequency domain Green's function of the problem allows the calculation of both the electric and the magnetic radiated fields. A novel analytical formula for the total radiated power of each multipactor harmonic has been derived. This formula is suitable for predicting multipactor with the third-harmonic technique. The proposed formulation has been successfully tested with a particle-in-cell code.
\end{abstract}

Index Terms-Microwave discharges, multipactor effect, parallel-plate waveguide, radiated power, third-harmonic detection technique.

\section{INTRODUCTION}

$\mathbf{T}$ HE MULTIPACTOR effect is a phenomenon of RF breakdown occurring in microwave components for space telecommunications subsystems [1] and accelerator structures [2] working under high-power conditions, which represents a possible failure mechanism. Multipactor dissipates power, loads cavities, degrades components, increases system noise level, and generates undesirable harmonics [3], [4]. As it is well known, multipactor occurs when a harmonic electric field exists in a parallel-plate region, which accelerates the electrons from one surface to the other. After impact, more electrons are released: If certain resonant conditions are satisfied, and the secondary-electron-yield coefficient is higher than unity, an electron avalanche is generated. Classical multipactor theory derives analytical expressions for such resonant conditions of the electron phases and voltages in which steady-state multipactor may exist [5]-[7]. In addition, multipactor has been

Manuscript received February 25, 2008. The review of this paper was arranged by Editor W. Menninger.

E. Sorolla and B. Gimeno are with the Departamento de Física Aplicada, Instituto de Ciencia de Materiales, Universidad de Valencia, 46100 Valencia, Spain (e-mail: benito.gimeno@uv.es).

S. Anza and J. Gil are with Aurora Software and Testing, S.L., Parque Científico de la Universidad de Valencia, 46980 Valencia, Spain.

A. M. Pérez, C. Vicente, and V. E. Boria are with the Departamento de Comunicaciones, Instituto de Telecomunicaciones y Aplicaciones Multimedia, Universidad Politécnica de Valencia, 46022 Valencia, Spain.

F. J. Pérez-Soler, F. D. Quesada, and A. Álvarez are with the Universidad Politécnica de Cartagena, 30202 Cartagena, Spain.

Digital Object Identifier 10.1109/TED.2008.926271 recently studied in more complex structures, such as the rectangular waveguide [8].

The main effort of previous works has been focused on the prediction of the multipactor threshold voltage, the study of the higher order resonant modes, the analysis of steadystable trajectories, and the study of multipaction detection methods. Also, different technical aspects, as the modeling of dielectric windows used in high-power RF systems, have been investigated [9]. However, the problem of the radiated power by a multipactor discharge has not been studied in detail. Such problem is very important from a practical point of view, because the noise created by a discharge is a method typically used to experimentally detect the phenomenon (third-harmonic detection technique) [4], [10], [11]. From a qualitative point of view, the total radiated power of an electron can be estimated by means of the Larmor formula, which states that such power is proportional to the square of the electron acceleration [4]. Larmor formula evaluates the total radiated power by an electron moving in free space, not considering the effect of the realistic parallel-plate scenario. Our main goal has been to calculate the total radiated power by a discharge in the context of the parallel-plates classical multipactor theory.

In this paper, we present a simple and novel model to evaluate the electric and magnetic radiated fields as well as the radiated power by a multipactor discharge excited in a parallel-plate region driven by an RF electric field. In this first approach to the problem, the discharge is modeled as a uniform electric current radiating inside an infinite parallel-plate waveguide region. The electromagnetic field radiated by the multipactor current has been evaluated using the frequency domain Green's function of the problem, which allows the direct calculation of the complex Poynting's vector of the radiated fields for each harmonic. Next, the total radiated power is expressed in terms of the most relevant parameters of the problem. Finally, comparisons with numerical results using a particle-in-cell (PIC) code validates this novel formulation.

\section{THEORY}

\section{A. Time-Harmonic Representation of the Multipactor Current}

For simplicity, we shall use a 1-D model where multipactor occurs inside a parallel-plate region of width $d$ driven by the RF voltage $V(t)=V_{\mathrm{RF}} \cos (\omega t)$, where $V_{\mathrm{RF}}$ is the voltage amplitude, $f=\omega /(2 \pi)$ is the RF frequency, and $t$ is the time; 


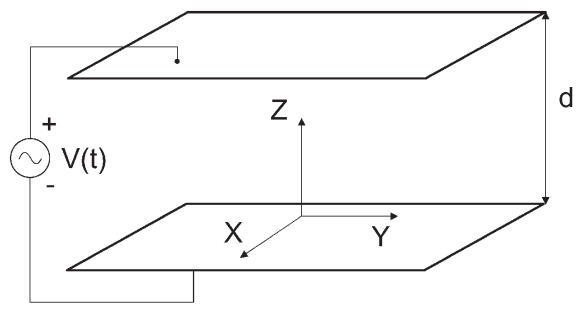

Fig. 1. Parallel-plate waveguide region driven by an RF voltage $V(t)$.

the period of this signal is $T=1 / f$, and the direction of motion is $z$ (see Fig. 1). The discharge is described using an effective electron model which operates in perfect multipactor resonant conditions: An electron launched from one plate is accelerated by a uniform electric field $\vec{E}=(V / d) \widehat{z}$ impacting to other plate in a transit time which is $N$ half RF periods ( $N$ is odd). We have considered that the initial velocity is constant in all launches, as stated in Sombrin's [12] model. Assuming that the electron is emitted at time $t_{\alpha}=\alpha / \omega$, the equation of motion (directly derived from the Lorentz force) can be analytically integrated by just obtaining both electron velocity $v_{e}(t)$ and electron position $z_{e}(t)$ as a function of time; obviously, $v_{e}=\left(d z_{e} / d t\right)$. Thus, by considering that the electron is launched from the plate situated in $z=0$, with initial velocity $v_{e}\left(t_{\alpha}\right)=v_{e_{0}}$, and operating under multipactor order $N=1,3,5 \ldots$, the electron velocity can be expressed as follows:

$$
v_{e}(t)=\left\{\begin{array}{c}
v_{e_{0}}+G(\sin (\alpha)-\sin (\omega t)) \\
t \in\left[t_{\alpha}, t_{\alpha}+\frac{N T}{2}\right] \\
-v_{e_{0}}-G(\sin (\alpha)+\sin (\omega t)) \\
t \in\left[t_{\alpha}+\frac{N T}{2}, t_{\alpha}+N T\right]
\end{array}\right.
$$

where $G=\left(e V_{\mathrm{RF}}\right) /\left(m_{e} \omega d\right)$, being $m_{e}$ and $-e$ the electron mass and charge, respectively. Note that the electron velocity is a periodic function of period $N T$. In this context, the multipactor RF breakdown voltage can be simply calculated as described in [6], resulting

$$
V_{\mathrm{RF}}=\frac{m_{e}}{e} \frac{\omega d\left(\omega d-N \pi v_{e_{0}}\right)}{N \pi \sin (\alpha)-2 \cos (\alpha)} .
$$

The multipactor current created by the effective electron $J_{z}$ can be expressed as a single current density which is function of the position $z$ and time $t$

$$
J_{z}(z, t)=-e v_{e} \delta\left(z-z_{e}(t)\right)
$$

where $\delta$ is the Dirac Delta function, $v_{e}$ is the electron velocity given in (1), and $z_{e}(t)$ is the electron position. For far-field observation points, we might consider that such multipactor electric current is homogeneously distributed throughout the gap. Thus, we define an equivalent multipactor thin wire current $I(t)$ as the spatial average of the current

$$
I(t) \equiv\left\langle J_{z}(z, t)\right\rangle=\frac{1}{d} \int_{0}^{d}-e v_{e} \delta\left(z-z_{e}(t)\right) d z=\frac{-e}{d} v_{e}(t) .
$$

In order to study its power radiation pattern, we consider that this current radiates in the parallel-plate region. Note that, although $v_{e}(t)$ in (3) is not a harmonic function, $I(t)$ is a periodic function of period $N T$, so we can expand it in terms of an infinite Fourier series

$$
I(t)=\frac{-e}{d} \sum_{m=1}^{+\infty} c_{m} \cos \left(m \frac{\omega}{N} t+\psi_{m}\right) .
$$

The Fourier coefficients $c_{m}$ and $\psi_{m}$ have been analytically calculated as $c_{m}=\sqrt{a_{m}^{2}+b_{m}^{2}}$ and $\psi_{m}=-\arctan \left(b_{m} / a_{m}\right)$, where

$$
\begin{aligned}
& a_{m}=\frac{-2\left(1-(-1)^{m}\right)\left(v_{e_{0}}+G \sin \alpha\right) \sin (m \alpha / N)}{m \pi} \\
& b_{m}=-G \delta_{m, N}+\frac{2\left(1-(-1)^{m}\right)\left(v_{e_{0}}+G \sin \alpha\right) \cos (m \alpha / N)}{m \pi \quad} \quad \\
& m=1,3,5, \ldots
\end{aligned}
$$

where $\delta_{m, N}$ is the Kronecker Delta function; note that for even values of $m$ both coefficients $a_{m}$ and $b_{m}$ are equal to zero. Following the standard complex notation for harmonic fields, we define the complex currents $i_{m}$ as

$$
i_{m} \equiv \frac{-e}{d} c_{m} \mathrm{e}^{j \psi_{m}}, \quad m=1,3,5, \ldots
$$

corresponding to the $m$ th Fourier harmonic of frequency $f_{m}=$ $m(f / N)$; thus, the instantaneous value of the equivalent multipactor current can be expressed as the real part of these complex forms

$$
I(t)=\Re\left(\sum_{m=1}^{+\infty} i_{m} e^{j m \frac{\omega}{N} t}\right) .
$$

At this point of the formulation, we can use the classical radiation theory to study the power radiated by the time-harmonic multipactor currents $i_{m}$ in the parallel-plate region.

\section{B. Radiation of the Time-Harmonic Multipactor Currents in the Parallel-Plate Waveguide}

We need to calculate the electromagnetic fields radiated by a uniform time-harmonic current of length $d$ and frequency $f_{m}$ placed in a parallel-plate region, as shown in Fig. 1; cylindrical coordinates will be used along this paper. In order to proceed, we will use the scalar and vectorial potentials formulation for time-harmonic fields in free-space, which is based on the calculation of both the electric scalar $(\phi)$ and the magnetic vector potentials $(\vec{A})$, satisfying the Lorentz gauge [13]. For the case of a current $i_{m}$ oriented in the $z$ axis, the vector potential will have only nonzero $z$ component, denoted as $A_{z_{m}}$, which satisfies the Helmholtz differential equation

$$
\nabla^{2} A_{z_{m}}+k_{m}^{2} A_{z_{m}}=-\mu_{0} i_{m}
$$

where $k_{m}=m(k / N)$ is the wavenumber of the mth Fourier harmonic, being $k=\omega / c$ the free-space wavenumber; note that $c$ is the speed of light in vacuum, and the form $e^{j m(\omega / N) t}$ 
(a)

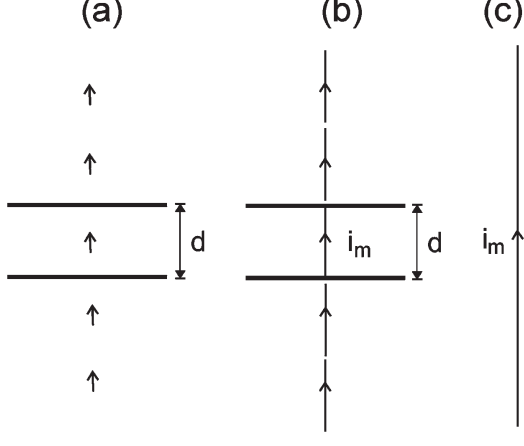

Fig. 2. Description of the multipactor current. (a) Infinite reflections of a point current between two parallel plates. (b) Uniform multipactor current is infinitely reflected in the parallel-plate region. (c) Multipactor current in the parallel-plate region is equivalent to an infinite wire uniform current.

has been omitted in the formulation. The solution of such equation can be expressed in integral form as a function of the $z$ component of the vector potential Green's function $g_{z}\left(\vec{r}, \vec{r}^{\prime}\right)$

$$
A_{z_{m}}(\vec{r})=\mu_{0} \int_{0}^{d} g_{z}\left(\vec{r}, \vec{r}^{\prime}\right) i_{m} d z^{\prime}
$$

with $\vec{r}^{\prime}=z^{\prime} \widehat{z}$. The Green's function satisfies the scalar Helmholtz equation

$$
\nabla^{2} g_{z}\left(\vec{r}, \vec{r}^{\prime}\right)+k^{2} g_{z}\left(\vec{r}, \vec{r}^{\prime}\right)=-\delta\left(\vec{r}-\vec{r}^{\prime}\right)
$$

with the Neumann boundary condition.

The next step in the formulation is to obtain such a Green's function in a closed form for an easy integration of (6). The response of a $z$-directed infinitesimal current located at the source point $\vec{r}^{\prime}=z^{\prime} \widehat{z}$ can be obtained using the classical theory of images [13]: The point current is successively reflected as an infinite images series in both metallic plates, as shown in Fig. 2(a). In this particular case, the multipactor current $i_{m}$ is spatially uniform, which makes possible to directly transform the wire current, infinitely reflected in both plates, into an infinite uniform current radiating in free space, as shown in Fig. 2(b). As a consequence, the finite current of length $d$, radiating between the two conducting parallel plates, might be replaced by an infinite wire carrying the constant current $i_{m}$ radiating in free space, as shown in Fig. 2(c). At this point, the well-known free space 3-D Green's function of the scalar Helmholtz equation $g_{0}\left(\vec{r}, \vec{r}^{\prime}\right)$ can be applied

$$
g_{0}\left(\vec{r}, \vec{r}^{\prime}\right)=\frac{e^{-j k R}}{4 \pi R}
$$

being $R$ the magnitude of the vector $\vec{R}(R \equiv\|\vec{R}\|)$, which is defined as $\vec{R} \equiv \vec{r}-\vec{r}^{\prime}$.

We now turn to proceed with the integration of (6), which is reduced to

$$
A_{z_{m}}(\vec{r})=\mu_{0} i_{m} \int_{-\infty}^{+\infty} g_{0}\left(\vec{r}, \vec{r}^{\prime}\right) d z^{\prime}
$$

where the field and source points are $\vec{r}=r \widehat{r}+z \widehat{z}$ and $\vec{r}^{\prime}=z^{\prime} \widehat{z}$, respectively. By substituting (7) in this integral, we obtain

$$
\begin{aligned}
A_{z_{m}}(\vec{r}) & =\frac{\mu_{0}}{4 \pi} i_{m} \int_{-\infty}^{+\infty} \frac{\exp \left(-j k_{m} \sqrt{r^{2}+\left(z-z^{\prime}\right)^{2}}\right)}{\sqrt{r^{2}+\left(z-z^{\prime}\right)^{2}}} d z^{\prime} \\
& =\frac{\mu_{0}}{4 \pi} i_{m}(-j) \pi H_{0}^{(2)}\left(k_{m} r\right) \\
& =\mu_{0} i_{m}\left(\frac{H_{0}^{(2)}\left(k_{m} r\right)}{4 j}\right)
\end{aligned}
$$

where $H_{0}^{(2)}$ is the zeroth-order Hankel function of the second kind. Note that the term between brackets is typically referred as the free-space 2-D Green's function, remarking that our original 3-D problem has been converted into a 2-D one. In fact, the dependence with the $z$-coordinate has consequently disappeared. Then, the Lorentz gauge dictates that the electric scalar potential $\phi_{m}$ vanishes

$$
\phi_{m}=j \frac{c}{k_{m}} \nabla \cdot\left(A_{z_{m}} \widehat{z}\right)=j \frac{c}{k_{m}} \frac{\partial A_{z_{m}}}{\partial z}=0 .
$$

The far-fields radiated by the multipactor current can now be easily evaluated utilizing the asymptotic expansion of $H_{0}^{(2)}$ for large arguments

$$
H_{0}^{(2)}(x) \approx \sqrt{\frac{2}{\pi x}} e^{-j(x-\pi / 4)}
$$

just obtaining

$$
\begin{aligned}
\vec{E}_{m} & =-j k_{m} c A_{z_{m}} \widehat{z} \approx-\eta i_{m} \sqrt{\frac{k_{m}}{8 \pi r}} e^{-j\left(k_{m} r-\pi / 4\right)} \widehat{z} \\
\vec{H}_{m} & =\frac{1}{\mu_{0}} \nabla \times\left(A_{z_{m}} \widehat{z}\right) \approx i_{m} \sqrt{\frac{k_{m}}{8 \pi r}} e^{-j\left(k_{m} r-\pi / 4\right)} \widehat{\varphi} .
\end{aligned}
$$

Note that higher order terms of $r^{-1 / 2}$ have been neglected; $\eta$ is the intrinsic impedance of the vacuum, $\eta=\sqrt{\mu_{0} / \varepsilon_{0}}$. To get a suitable expression for the power radiated by the multipactor current in the parallel-plate region, we calculate the complex Poynting's vector of each Fourier harmonic

$$
\vec{N}_{m} \equiv \frac{1}{2} \vec{E}_{m} \times \vec{H}_{m}^{*}=\eta\left|i_{m}\right|^{2} \frac{k_{m}}{16 \pi r} \widehat{r}
$$

where $*$ indicates complex conjugate. We remark that this vector only has radial component, which implies a net flow of energy in the radial direction. The final objective of this paper is to find such energy. The integration of the real part of the Poynting's vector in a cylinder of radius $r$ and height $d$ centered in the $z$ axis, allows to get a simple formula for the total power radiated by the $m$ th multipactor current, thus obtaining

$$
P_{m}=\frac{\pi}{4} \mu_{0} m \frac{f}{N} d\left|i_{m}\right|^{2}, \quad m=1,3,5, \ldots
$$

Inserting (5) into (11) and using the value of the multipactor RF breakdown voltage (2), we have found, after simple algebraic 
manipulations, the following formula:

$$
P_{m}=\frac{\omega \mu_{0} m e^{2}}{8 N d}\left[\delta_{m, N}\left(G^{2}-\frac{8 U Q(-2+W S)}{m \pi}\right)+\left(\frac{4 U}{m \pi}\right)^{2}\right]
$$

where the terms $Q, S, T$, and $U$ have been defined as follows:

$$
\begin{aligned}
Q & \equiv \frac{\omega d-N \pi v_{e_{0}}}{4+N^{2} \pi^{2}} \\
S & \equiv \sqrt{\frac{e^{2} V_{\mathrm{RF}}^{2}\left(4+N^{2} \pi^{2}\right)}{\left(\omega d-N \pi v_{e_{0}}\right)^{2}}-\left(m_{e} \omega d\right)^{2}} \\
T & \equiv N \pi+\frac{2 S}{\omega d m_{e}} \\
U & \equiv v_{e_{0}}+Q T \\
W & \equiv \frac{N \pi}{\omega d m_{e}} .
\end{aligned}
$$

At this point, it is important to understand that (12) gives the power spectrum for a single electron, according to the proposed theory. However, since the multipactor current is directly proportional to the number of electrons involved in the multipactor discharge (5), the power emitted by $n_{e}$ electrons in resonance would be simply given by $n_{e}^{2} P_{m}$, thus being (12) universal and valid for any electron density. On the other hand, we want to emphasize that the radiated power by multipactor occurs at odd multiples of $f / N$, which means that harmonics will be generated at lower frequencies than the RF signal frequency $f$ for multipactor orders $N>1$. Moreover, the first term of (12), which is multiplied by $\delta_{m, N}$, will be different from zero when $m=N$, i.e., for the harmonic located at the RF frequency. For the rest of the harmonics, the formula predicts a decreasing power level with harmonic order following a $1 / \mathrm{m}$ behavior. Another interesting result is that (12) breaks up the traditional dependence of all multipactor parameters related with the prediction of the RF threshold voltage on the $\omega d$ product [5], [6]. In fact, the power level depends on $\omega / d$ for all the harmonics, if working with a fixed $\omega d$. This implies, for instance, that the predicted harmonic levels for real low band waveguides (low frequency, large gap) would be lower than for high band ones, even if working with the same $\omega d$. In addition, multipactor in typical large gap structures, such as bandpass filters, would considerably generate lower harmonics than other structures with shorter gaps, such as transformers or low-pass filters. Such implications should be also considered when measuring multipactor in real devices.

\section{RESULTS}

First, we present results for the convergence of the timeharmonic representation of the multipactor current. In Fig. 3, such a current is plotted as a function of the normalized time $t / T$; these numerical results have been computed with both the exact (3) and the Fourier series (4) for multipactor order $N=3$. Different number of odd terms have been considered in the summation, demonstrating that we need at least 200 odd harmonics to reach the convergence region. The data employed for

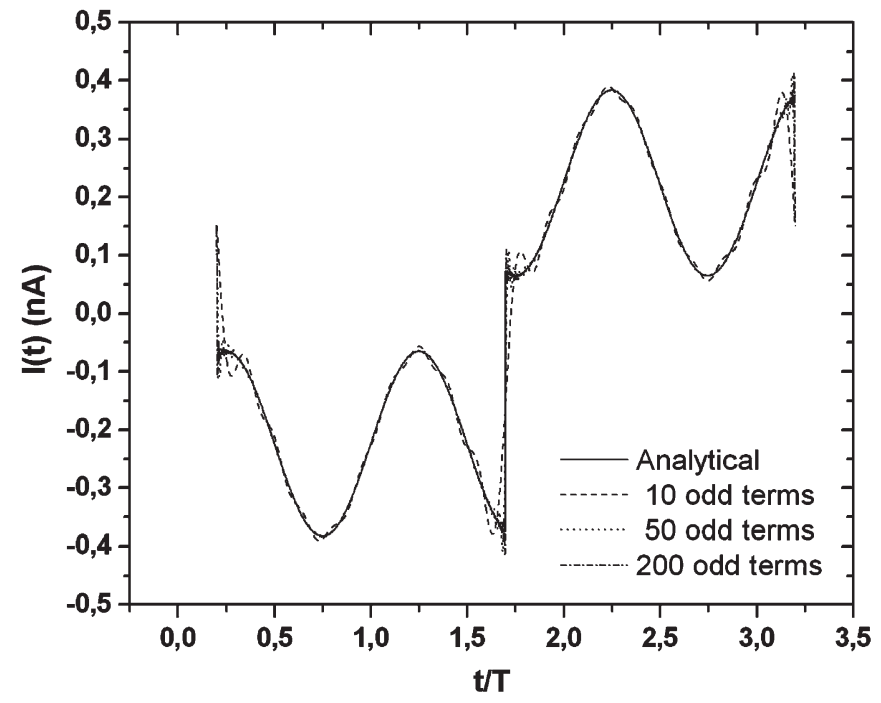

Fig. 3. Multipactor current as a function of the normalized time $t / T$ for order $N=3$. Comparison between the analytical expression (3) and the Fourier expansions evaluated with different number of odd terms (4).

these simulations in order to get perfect resonance conditions are the following: $f=2 \mathrm{GHz}, d=2.5 \mathrm{~mm}, v_{e_{0}}=3.68 \mathrm{eV}$, $V_{\mathrm{RF}}=442.9 \mathrm{~V}, \alpha=72^{\circ}$.

In Fig. 4, the total radiated power of a multipactor current obtained with (12) is successfully compared to simulations performed with an electromagnetic PIC code based on a finitedifference in time-domain solver [14]. In order to check the presented algorithm, four cases have been analyzed for multipactor orders $N=1,3,5,11$. In the four cases, the RF frequency was $f=1 \mathrm{GHz}$, and the initial speed is $v_{e_{0}}=3.68 \mathrm{eV}$. Other relevant parameters in the simulations are the following: For $N=1$, we used $d=1 \mathrm{~mm}, \alpha=92.49^{\circ}, V_{\mathrm{RF}}=30 \mathrm{~V}$; for $N=3$, we used $d=5 \mathrm{~mm}, \alpha=101.96^{\circ}, V_{\mathrm{RF}}=383.64 \mathrm{~V}$; for $N=5$, we used $d=7 \mathrm{~mm}, \alpha=97.15^{\circ}, V_{\mathrm{RF}}=412.34 \mathrm{~V}$; for $N=11$, we used $d=15.65 \mathrm{~mm}, \alpha=93.30^{\circ}, V_{\mathrm{RF}}=$ $953.14 \mathrm{~V}$. The numerical results match perfectly the analytical predictions for both harmonic frequency and power level. The power levels of the results are extremely low (on the order of $-200 \mathrm{~dB} \cdot \mathrm{W})$ as corresponds to the emission of a single electron. In fact, as stated before, in real situations, the multipactor avalanche consists of several resonant electrons $\left(n_{e}\right)$ that would increase the presented nominal power by a factor $n_{e}^{2}$. For instance, for a typical avalanche of, for example, $n_{e}=10^{8}$ electrons, all the curves in Fig. 4 would be shifted $+160 \mathrm{~dB}$ upwards. These results verify, as predicted by (12), that the power level of the harmonics generated by a multipactor discharge decreases as $1 / m$ for all the harmonics orders $m$ except for the $m=N$ order, in which the maximum power is emitted.

Finally, we want to make some remarks on the thirdharmonic technique for multipactor detection [4], [11]. In the case of multipactor order $N=1$, the mentioned third harmonic coincides indeed with the $m=3$ harmonic, being detected at a frequency three times higher than the RF one. As it is well known, this is due to the fact that this harmonic is the one with highest level that appears for all the multipactor orders (apart from the main one at the RF frequency $f$, which is obscured by the carrier in an experiment). However, for higher order 

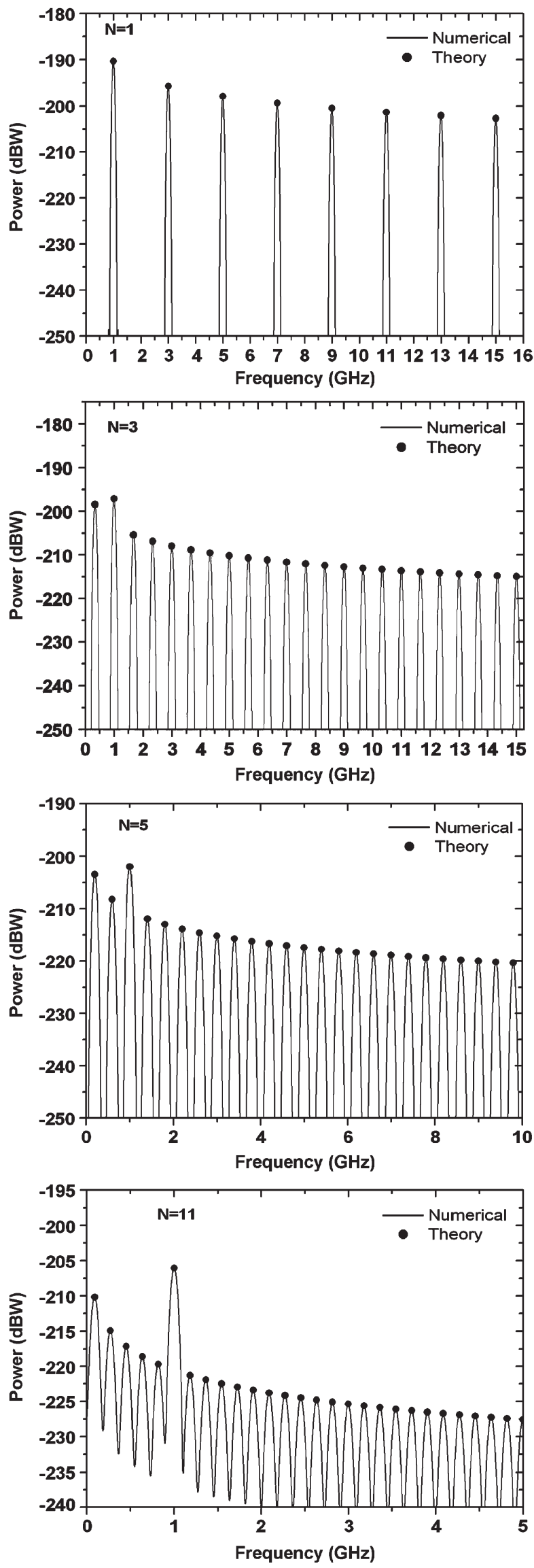

Fig. 4. Power spectrum generated by a multipactor discharge for different multipactor orders: $N=1, N=3, N=5$ and $N=11$. multipactor orders $N>1$, more harmonics appear to both sides of the RF carrier, at odd multiples of $f / N$, whose amplitudes are higher than the third-harmonic one (see Fig. 4). In the cases in which such harmonics are propagated and are not obscured by the carrier, they could give a more precise reference for detecting multipactor.

\section{CONCLUSION}

In this paper, we present an analytic model to calculate the total radiated power by a multipactor current occurring in a parallel-plate waveguide region. The proposed formulation is based on the time-harmonic representation of the multipactor discharge current. A closed-form expression for the radiated power of each harmonic involving the relevant parameters of the phenomenon has been derived in detail. In order to check such expression, comparisons to numerical results obtained with a PIC code have been shown, obtaining excellent agreement, and fully validating the presented approach. The formulation derived might be easily used to predict the full radiated spectrum of a multipactor discharge, helping to correlate theoretical predictions with experimental results, and ultimately giving a more precise reference for multipactor detection.

\section{REFERENCES}

[1] J. de Lara, F. Pérez, M. Alfonseca, L. Galán, I. Montero, E. Román, and D. Raboso, "Multipactor prediction for on-board spacecraft RF equipment with the MEST software tool," IEEE Trans. Plasma Sci., vol. 34, no. 2, pp. 476-484, Apr. 2006.

[2] T. Abe, T. Kageyama, K. Akai, K. Ebihara, H. Sakai, and Y. Takeuchi, "Multipactoring zone map of an RF input coupler and its application to high beam current storage rings," Phys. Rev. Special Topics-Accelerators Beams, vol. 9, no. 6, p. $062002,2006$.

[3] R. Kishek and Y. Y. Lau, "Interaction of multipactor discharge and RF circuit," Phys. Rev. Lett., vol. 75, no. 6, pp. 1218-1221, Aug. 1995.

[4] R. Udiljak, D. Anderson, P. Ingvarson, U. Jordan, U. Jostell, L. Lapierre, G. Li, M. Lisak, J. Puech, and J. Sombrin, "New method for detection of multipaction," IEEE Trans. Plasma Sci., vol. 31, no. 3, pp. 396-404, Jun. 2003.

[5] A. J. Hatch and H. B. Williams, "Multipacting modes of high-frequency gaseous breakdown," Phys. Rev., vol. 112, no. 3, pp. 681-685, Nov. 1958.

[6] J. R. M. Vaughan, "Multipactor," IEEE Trans. Electron Devices, vol. 35, no. 7, pp. 1172-1180, Jul. 1988.

[7] N. K. Vdovicheva, A. G. Sazontov, and V. E. Semenov, "Statistical theory of two-sided multipactor," Radiophys. Quantum Electron., vol. 47, no. 8, pp. 580-596, Aug. 2004.

[8] V. E. Semenov, E. I. Rakova, D. Anderson, M. Lisak, and J. Puech, "Multipactor in rectangular waveguides," Phys. Plasmas, vol. 14, no. 3, p. 033 501, Mar. 2007.

[9] H. C. Kim, J. P. Verboncoeur, and Y. Y. Lau, "Modeling RF window breakdown: From vacuum multipactor to RF plasma," IEEE Trans. Dielectr. Electr. Insul., vol. 14, no. 4, pp. 774-782, Aug. 2007.

[10] Space Engineering: Multipacting Design and Test, ESA Publication Division, Noordwijk, The Netherlands, May 2003. ECSS-20-01A, edited by ESA-ESTEC

[11] A. J. Marrison, R. May, J. D. Sanders, A. D. Dyne, A. D. Rawlins, and J. Petit, A study of multipaction in multicarrier RF components, Jan. 1997, Culham, U.K.. AEA Technology for ESA/ESTEC, Ref. AEA/TYKB/ 31761/01/RP/05.

[12] J. Sombrin, "Effet multipactor," CNES, Toulouse, France, CNES Tech. Rep. No. 83/DRT/TIT/HY/119/T, 1983.

[13] C. A. Balanis, Advanced Engineering Electromagnetics. New York: Wiley, 1989.

[14] S. Anza, C. Vicente, D. Raboso, J. Gil, B. Gimeno, and V. E. Boria, "Enhanced prediction of multipaction breakdown in passive waveguide components including space charge effects," in Proc. IEEE Int. Microw. Symp., Atlanta, GA, Jun. 2008. to be published. 


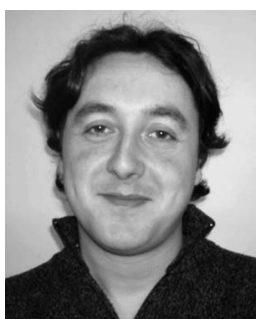

Edén Sorolla was born in Alicante, Spain, on May 12, 1980. He received the Licenciado degree in physics from the Universidad de Valencia, Valencia, Spain, in 2007.

$\mathrm{He}$ is currently with the Universidad de Valencia. From 2007 to 2008, he obtained a fellowship from the Departamento de Física Aplicada y Electromagnetismo and Instituto de Ciencia de Materiales, Universidad de Valencia. His current research interest includes nonlinear phenomena appearing in power microwave subsystems (multipactor effect, corona effects, and passive intermodulation phenomena).

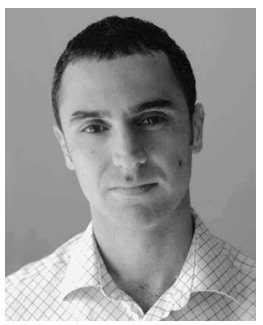

Sergio Anza was born in Madrid, Spain, in 1978. $\mathrm{He}$ received the B.S. degree in telecommunications engineering from the Universidad Politecnica de Valencia, Valencia, Spain, in 2002, and the M.S. degree in aerospace science and technology from the Universitat Politecnica de Catalunya, Barcelona, Spain, in 2006

$\mathrm{He}$ is currently with Aurora Software and Testing, S.L., Parque Científico de la Universidad de Valencia. During 2001-2002, he was a Visiting Scholar with the University of Bologna, Bologna, Italy. From 2003 to 2006, he was with the Consejo Superior de Investigaciones Cientificas of the Spanish Ministry of Science. During that period, he was with the Lisa Path Finder mission of the European Space Agency and the Global Navigation Satellite System Receiver for Atmospheric Sounding Satellite Application Facilities project. His current research interests include the areas of numerical techniques for the modeling and prediction of nonlinear phenomena in RF high-power devices for space applications, with especial emphasis on the study of the multipactor effect appearing in power microwave subsystems.

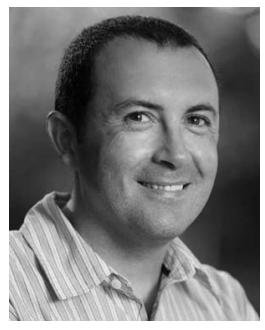

Benito Gimeno (M'01) was born in Valencia, Spain, on January 29, 1964. He received the Licenciado degree in physics and the Ph.D. degree from the Universidad de Valencia, Valencia, Spain, in 1987 and 1992, respectively.

He was a Fellow at the Universidad de Valencia from 1987 to 1990. In 1990, he served as an Assistant Professor with the Departamento de Física Aplicada y Electromagnetismo and Instituto de Ciencia de Materiales, Universidad de Valencia, where he became Associate Professor in 1997. He was working at European Space Research and Technology Centre of the European Space Agency as a Research Fellow during 1994 and 1995. In 2003, he obtained a fellowship from the Spanish Government for a short stay (three months) at the Universita degli Studi di Pavia, Pavia, Italy, as a Visiting Scientific. His current research interests include the areas of computer-aided techniques for analysis of microwave and millimeter-wave passive components for space applications, waveguides, and cavities structures including dielectric objects, electromagnetic band-gap structures, frequency selective surfaces, and nonlinear phenomena appearing in power microwave subsystems (multipactor effect, corona effects, and passive intermodulation phenomena).

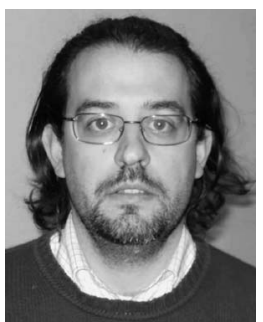

Antonio M. Pérez was born in Valencia, Spain, on May 7, 1977. He received the Licenciado degree in physics from the Universidad de Valencia, Valencia, in 2001.

Since 2005, he has been a Researcher with the Departamento de Comunicaciones, Universidad Politécnica de Valencia, Valencia. In 2003, he obtained a fellowship from the European Union for a one-year stage at the Universita degli Studi di Pavia, Pavia, Italy, as a Researcher. His current research interests include the areas of computer-aided techniques for analysis of microwave and millimeter-wave passive components for space applications, waveguides, and cavities structures including dielectric objects and nonlinear phenomena appearing in power microwave subsystems (multipactor effect).

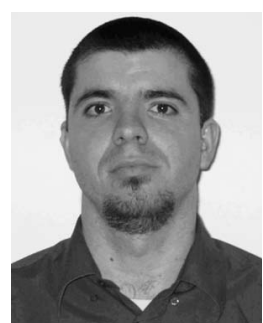

Carlos Vicente was born in Elche, Spain, in 1976. He received the Dipl. degree in physics from the University of Valencia, Valencia, Spain, in 1999, and the Dr.Ing. degree in engineering from the Technical University of Darmstadt, Darmstadt, Germany, in 2005 .

From 1999 to the beginning of 2001, he worked as a Research Assistant with the Department of Theoretical Physics, University of Valencia. From 2001 to 2005, he was a Professor Assistant with the Institute of Microwave Engineering, Technical University of Darmstadt. Since 2005, he has been with the Departamento de Comunicaciones, Instituto de Telecomunicaciones y Aplicaciones Multimedia, Universidad Politécnica de Valencia, Valencia. In 2006, he cofounded the Aurora Software and Testing, S.L. devoted to the telecommunications sector. His research concerns passive intermodulation, corona discharge, and multipaction in communications satellite applications.

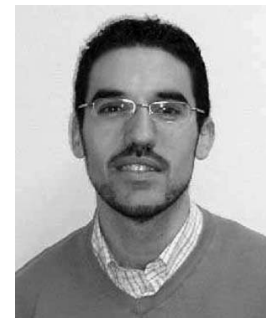

Jordi Gil was born in Valencia, Spain, on April 27 1977. He received the Licenciado degree in physics from the University of Valencia, in 2000, and the Master Philosophy Diploma in telecommunications engineering.

He is currently with Aurora Software and Testing, S.L., Parque Científico de la Universidad de Valencia. From 2001 to 2004, he worked as Researcher with an Aerospatiale Italian Company in the frame of the V European Framework Programme. From 2004 to 2006, he continued his research work at the Technical University of Valencia. In 2006, he cofunded the Company Aurora Software and Testing, S.L. devoted to the space sector. His current research interests include numerical methods in computer-aided techniques for the analysis of microwave and millimeter passive components based on waveguide technology, and nonlinear phenomena appearing in power microwave subsystems for space applications.

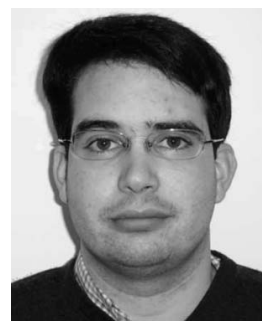

Francisco J. Pérez-Soler (S'05) was born in Murcia, Spain, on January 16, 1981. He received the Electrical Engineering degree from the Technical University of Cartagena, Cartagena, Spain, in 2004, where has been working toward the Ph.D. degree in the Communications and Information Technologies Department.

Since 2007, he has been an Assistant Professor for the same department. His current research interests include the areas of integral equation methods and fast numerical techniques for analysis of microwave components, waveguides, and cavity structures including dielectric objects.

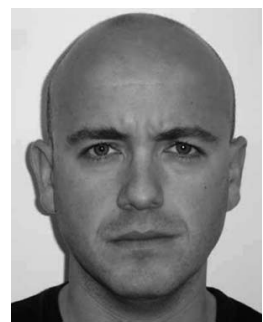

Fernando D. Quesada (S'05-M'07) was born in Murcia, Spain, in 1974. He received the Telecommunications Engineer degree from the Technical University of Valencia (UPV), Valencia, Spain, in 2000, and the Ph.D. degree from the Universidad Politécnica de Cartagena (UPCT), Cartagena, Spain, in 2007.

He is currently with UPCT. In 1999, he was with the Radiocommunications Department, UPV, as a Research Assistant, where he was involved in the development of numerical methods for the analysis of anechoic chambers and tag antennas. In 2001, he was with the Communications and Information Technologies Department, UPCT, initially as a Research Assistant, and then as an Assistant Professor. In 2005, he spent six months as a Visiting Scientist with the University of Pavia, Pavia, Italy. His current scientific interests include IE numerical methods for the analysis of antennas and microwave devices. 


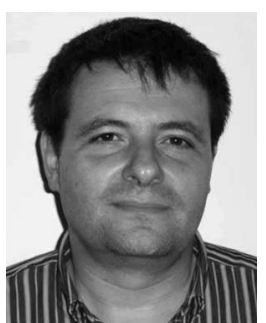

Alejandro Álvarez (M'99-SM'07) was born in Madrid, Spain, in 1965. He received the Telecommunications Engineer degree from the Technical University of Madrid (UPM), Madrid, in 1991, and the Ph.D. degree in electrical engineering from the Swiss Federal Institute of Technology, Lausanne, Switzerland, in 1998.

In 1988, he was with the Signal, Systems and Radiocommunications Department, UPM, as a Research Student, where he was involved in the design, testing, and measurement of broadband spiral antennas for electromagnetic measurements support equipment. From 1991 to 1993, he was with the Radio Frequency Systems Division, European Space Agency (ESA)/European Space Research and Technology Centre (ESTEC), Noordwijk, The Netherlands, where he was involved in the development of analytical and numerical tools for the study of waveguide discontinuities, planar transmission lines, and microwave filters. From 1993 to 1995, he was with the Space Division, Industry Alcatel Espacio, Madrid, and was also with the ESA, where he collaborated in several ESA/ESTEC contracts. From 1995 to 1999, he was with the Swiss Federal Institute of Technology, Ecole Polytechnique Fédérale de Lausanne, Lausanne, where he was involved with the field of microstrip antennas and printed circuits for space applications. In 2000, he was with the Universidad Politécnica de Cartagena, Cartagena, Spain, where he is currently developing his teaching and research activities.

Dr. Alvarez was the recipient of the Journèe Internationales de Nice Sur les Antennes (JINA) Best Paper Award for the best contribution to the JINA'98 International Symposium on Antennas, and the Colegio Oficial de Ingenieros de Telecomunicación Award to the best Ph.D. thesis in basic information and communication technologies.

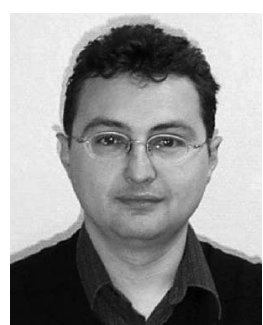

Vicente E. Boria (S'91-A'99-SM'02) was born in Valencia, Spain, on May 18, 1970. He received the "Ingeniero de Telecomunicación" degree (with first-class honors) and the "Doctor Ingeniero de Telecomunicación" degree from the Universidad Politécnica de Valencia, Valencia, Spain, in 1993 and 1997, respectively.

In 1993, he was with the "Departamento de Comunicaciones," Universidad Politécnica de Valencia, where he served as an Assistant Lecturer (19931995), Lecturer (1996-1997), and Associate Professor (1998-2002), and currently, he has been a Full Professor since 2003. In 1995 and 1996, he was holding a Spanish Trainee position with the European Space Research and Technology Centre, European Space Agency, Noordwijk, The Netherlands, where he was involved in the area of electromagnetic analysis and design of passive waveguide devices. He has authored or coauthored five chapters in technical textbooks, 50 papers in refereed international technical journals, and over 150 papers in international conference proceedings. His current research interests are focused on the analysis and automated design of passive components, left-handed and periodic structures, as well as on the simulation and measurement of power effects in passive waveguide systems.

Dr. Boria has been a member of the IEEE Microwave Theory and Techniques (IEEE-MTT) Society and the IEEE Antennas and Propagation Society since 1992. He is member of the Editorial Boards of the IEEE TRANSACTIONS ON Microwave THEORY AND TeChNiQues, IEEE MicrowaVe AND Wireless Components LetTers, Proceeding of the IEE (Microwaves, Antennas and Propagation), IEE Electronics Letters and Radio Science. He is also a member of the Technical Committees of the IEEE-MTT International Microwave Symposium and of the European Microwave Conference. He was the recipient of the 1993 First National Prize of Telecommunication Engineering Studies ("Ministerio de Educación y Ciencia"). He was also the recipient of the 2001 Social Council of Universidad Politécnica de Valencia First Research Prize for his outstanding activity during the period 1995-2000. 